\title{
Depression during Pregnancy and Postpartum Period among Women
}

\author{
Ms. Puja Kumari ${ }^{1 *}$, Dr. Gayatri Raina ${ }^{2}$
}

\section{ABSTRACT}

Depression during pregnancy and postpartum period affect women drastically. The present work evaluated depression during pregnancy and postpartum period among women of Shimla town, Himachal Pradesh. Eighty women were contacted during their third trimester of pregnancy and again during their one month postpartum period. Depression was assessed with Zung SelfRating Depression Scale. t-Test was applied to see the difference between the level of depression during third trimester of pregnancy and postpartum period. The result revealed that depression was higher during third trimester of pregnancy as compared to postpartum period among women although the difference failed to reach the level of significance.

Keywords: Depression, Pregnancy, Postpartum Period

Depression is a state of despondency marked by feelings of sadness, powerlessness and hopelessness. Emotional disorders primarily involving sadness, low mental or physical activity, come under depressive state. People use to have minor bouts of depression commonly, which is why depression is also known as common cold of psychopathology (Seligman, 1973). It is thought that females are at least twice more at risk than males. During the age period from 25-45, married women have particularly high rate of depression. While unmarried in this time bracket have much lower rate which is more similar to men (Paykel, 1991). This difference may reflect the greater stress for married women from both heavy child-care responsibilities and limited support provision from extended families, in addition to job stress. It is observed that the onset of depressive symptoms during pregnancy can occur at any stage of pregnancy (Klerman, 2006). The prevalence range of depression falls between 10-25 per cent during pregnancy and postpartum period (Goyal, Gay and Lee, 2010).Comparing the level of depression during

\footnotetext{
${ }^{1}$ Research Scholar, Department of Psychology, HP University, Summer Hills, Shimla, Himachal Pradesh, India

${ }^{2}$ Assistant Professor, Department of Psychology, HP University, Summer Hills, Shimla, H.P., Himachal Pradesh, India

*Responding Author
}

Received: December 30, 2016; Revision Received: January 20, 2017; Accepted: January 30, 2017

(C) 2017 Kumari P, Raina G; licensee IJIP. This is an Open Access Research distributed under the terms of the Creative Commons Attribution License (www.creativecommons.org/licenses/by/2.0), which permits unrestricted use, distribution, and reproduction in any Medium, provided the original work is properly cited. 


\section{Depression during Pregnancy and Postpartum Period among Women}

pregnancy and postpartum period, some studies reported that depression level was higher after delivery than during pregnancy (O’Hara, 1986).

O’Hara (1986) tried to reveal the relationship between social support, life events and depression during pregnancy and puerperium. They conducted a study on 99 women from second trimester to nine months postpartum. Depressed and non- depressed women were identified at second trimester. The 9 weeks postpartum assessment were compared on stressful life events and social support provided by their spouse and close confidents. The results indicated that 9 and 12 per cent women were found depressed during pregnancy and postpartum period, respectively. The study revealed that women who had postpartum depression reported more stressful life events and less support from their spouses after delivery than the women who did not have postpartum depression. Moreover, women experiencing depression during pregnancy reported somewhat less support from their spouses and more support from their confidants than non-depressed women.

Some research works suggested that there was no difference in the level of depression during pregnancy and postpartum period (Fisher et al., 2012; Mina et al. (2012). In India Mina, Balhara, Verma and Mathur (2012) assessed and compared the prevalence of depression during and after pregnancy in a hospital in Delhi. The total sample of 100 women was divided into two groups: 59 pregnant women in last trimester awaiting delivery and 41 women in postpartum period (4-12 weeks after childbirth). The two were compared on socio-demographic parameters. The results showed that both groups scored equally on depression scale during and after pregnancy.

On the other hand some researchers advocated that women reported a higher level of depressive symptomology during pregnancy as compared to post-delivery. Anderson in 2004 studied the implications of psychiatric disorders during pregnancy and the postpartum period. They estimated the point prevalence of mood, anxiety and eating disorders, based on DSM-IV criteria, among 1, 555 women attending ultrasound screening during the second trimester of pregnancy. They further assessed the obstetric and neonatal outcome, as well as the health care consumption during pregnancy, delivery and the early postpartum period among women with a psychiatric disorder, compared to healthy subjects in Sweden. The same procedure was made in a control group, consisting of 500 women, randomly selected among those who did not have any psychiatric diagnosis during the second trimester of pregnancy. The results revealed that out of the total of 1, 555 women, 220 had one or more prime-mood disorder diagnoses. Living single, low socioeconomic status, smoking, multi-parity and a body mass index of 30 or more were significantly associated with a psychiatric diagnosis in the second trimester of pregnancy. Women with antenatal depression and/or anxiety more often suffered from nausea and vomiting during pregnancy were more often on sick leave. Moreover, they visited their obstetrician more often than healthy subjects, specifically because of fear of childbirth and premature contractions. Also, they were more commonly delivered by elective caesarean section. These women had an increased use of epidural analgesia. The women reported a longer self -experienced duration of 


\section{Depression during Pregnancy and Postpartum Period among Women}

labour. The study further evidenced that severe complications of pregnancy, delivery, and the early postpartum period were not affected by antenatal depression and/or anxiety. There was no significant difference in neonatal outcome depending on antenatal depression or anxiety disorder. Though, fewer cases of depressive and/or anxiety disorders were prevalent during postpartum. Nonetheless, there was a significant shift from a majority of sub-threshold diagnoses during pregnancy to full DSM-IV diagnoses during the postpartum period. Moreover, previous psychiatric disorder and living singly were significantly associated with both a new-onset and a postpartum continuation/ recurrence of depression and/ or anxiety. Postpartum continuation/recurrence of a psychiatric disorder was additionally associated with smoking, obesity, and adverse obstetric events. Gebuza, Kazmierczak, Mieczkowska, Gierszewska and Kotzback (2014) focused on life satisfaction and social support received by women in the perinatal period and correlates of life satisfaction. The research sample included a total of 199 women in the third trimester of pregnancy and out of these women, 188 women were reobserved, who had physiological births or caesarean sections, during postpartum, in Bydgoszcz. The results clearly showed a significant increase in life satisfaction in the postpartum period among women. An important correlate of life satisfaction in the third trimester of pregnancy was social support received. During pregnancy such a correlate was emotional support received, and in the postnatal period- instrumental support (care and settling issue) received. The study found that the types and the time of social support received by women during pregnancy and postpartum period were significantly correlated with life satisfaction.

Curzik and Belgic (2012), observed 60 health prima parous women during late stage of pregnancy and two months postpartum in Zagreb, Croatia to explore the structure and intensity of depression and its relation to labour pain. Assessment of maximum and average labour pain was completed in three different time periods: before-expected labour pain, during/ immediately after labour- perceived labour pain and one month postpartum- recalled labour pain. The result analyses revealed that depressive symptoms reported in two months postpartum were significantly lower than the ones reported during late stage of pregnancy and was correlated significantly with labour pain expectancies.

From the above studies, it can be inferred that depression is prevalent and lowers the health status of women during pregnancy and postpartum period. This develops an urgent need to investigate the level of depression among women during these time period. The present research work was to scrutinize this need and designed to compare the level of depression among women during pregnancy and post- delivery.

\section{Objective:}

1. To compare depression level during third trimester of pregnancy and postpartum period among women. 


\section{Depression during Pregnancy and Postpartum Period among Women}

\section{Sample and Procedure}

Purposive sampling was done for collecting data for the present investigation. The present study consisted of 80 pregnant women from Shimla town, Himachal Pradesh. These women were approached during their third trimester of their pregnancies and were again contacted during their early postpartum period, i.e., 20- 30 days after delivery.

\section{Tool Used}

Zung Self-rating Depression Scale: Zung Self- rating Depression Scale (ZSDS) developed by Zung (1965) to fill the need for assessing depression, whether an effect, a symptom or a disorder, was chosen because of its accepted clinical value. It covers a broad range of depressive symptomology, dealing with the areas of pervasive affect, physiological equivalents and psychological concomitants (Zung, 1965). The scale is short and convenient. It consists of 20 items which are to be rated on a 4 point Likert scale. The self-report index covers, in varying degree; a broader spectrum including psychological, affective cognitive, behavioural and somatic aspects of depression.

Reliability and Validity: Knight et al. (1983) assessed the internal consistency of ZSDS, using alpha coefficient. It is found 0.79 which indicates a satisfactory level of homogeneity. It has well established reliability, replicability and validity (Zung, 1965). Upmanyu and Reen (1991) in an Indian sample of employed and unemployed females, found alpha coefficient for ZSDS to be 0.84. ZSDS is a valid and sensitive measure of clinical severity in depressed patients.

\section{Statistical Analysis}

t- Testwas applied to see the difference between the depression level during the two time periods, i.e., third trimester of pregnancy and postpartum period.

\section{RESULTS AND DISCUSSION}

Table 1: Mean, SD and t- Value between Third Trimester of Pregnancy and Postpartum Period on Depression Scores

\begin{tabular}{|l|l|l|l|l|}
\hline \multicolumn{2}{|l|}{ Third Trimester of Pregnancy } & \multicolumn{2}{l|}{ Postpartum Period } & \multirow{2}{*}{ t- Value } \\
\hline Mean & SD & Mean & SD & \\
\hline 39.75 & 6.78 & 33.83 & 6.80 & 5.52 \\
\hline
\end{tabular}

${ }^{* *} \mathrm{p}<.01,{ }^{*} \mathrm{p}<.05$ 
Depression during Pregnancy and Postpartum Period among Women

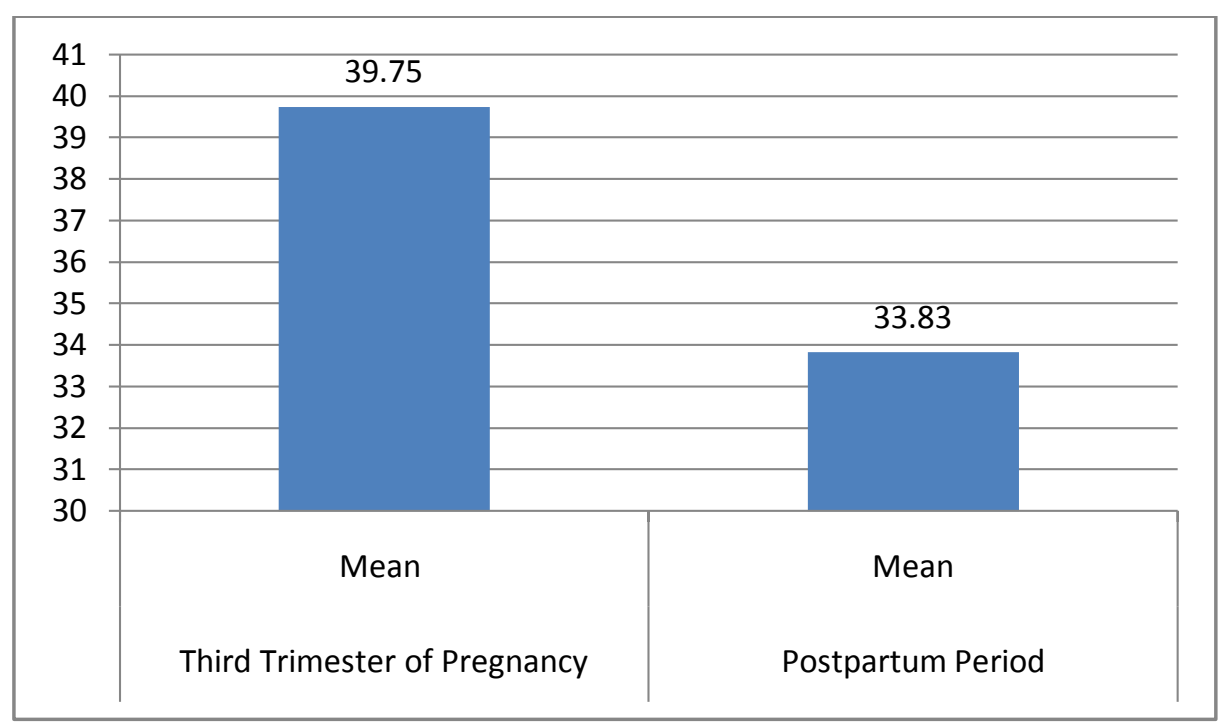

Figure1: Means of Depression Scores during Third Trimester of Pregnancy and Postpartum Period

Table 1 presents the mean, SD and t- value of third trimester of pregnancy and postpartum period for depression score. It is evident from Table 1 and from the graphical representation (Figure 1) that the mean of third trimester of pregnancy and postpartum period is 39.75 and 33.83, respectively. The t- value is 5.52 which did not reach the level of significance. This indicated that women experienced higher level of depression during third trimester of pregnancy as compared to postpartum period but not significantly.

Similar findings were reported by some researchers in their respective studies. Otchet et al. (1999) reported that women suffer more form emotional problem during pregnancy than postpartum period. Gebuza et al. (2014) observed that women's life satisfaction increased from pregnancy to postpartum period.

Further, Fisher et al. (2012) and Mina et al. (2012) in their respective studies reported that the frequency and level of depression among women remained same during late pregnancy and early postpartum period. But there are some contrary findings which indicate that women suffer from higher level of depression during postpartum period than pregnancy. O’Hara (1986) reported that women reported more stressful life events after delivery as compared to pregnancy.

Labour pain expectancies during late pregnancy and before the child birth may be the major factor of the higher level of depression during third trimester as compared to postpartum period among women. Anderson (2004) also argued that fear of child birth and apprehension of labour pain increased depressive symptoms during pregnancy which resulted in poor psychological well-being of the women. Similar findings were reported by Curzik et al. (2012) that depressive

(C) The International Journal of Indian Psychology, ISSN 2348-5396 (e)| ISSN: 2349-3429 (p) | 163 


\section{Depression during Pregnancy and Postpartum Period among Women}

symptoms highlighted during postpartum period were significantly lower than the ones reported during later stages of pregnancy in relation to labour pain.

\section{CONCLUSION}

The study found that women were at higher risk of depression during third trimester of pregnancy as compared to postpartum period but the difference between the levels of depression during these time periods did not reach at the level of significance. Though, the study underscores the need to direct the attention towards health risk in term of depression during pregnancy and postpartum period but it has its own limitation in term of small size of sample. Future studies in this area are suggested to investigate the level of depression on the bigger size of sample.

\section{Acknowledgments}

The author appreciates all those who participated in the study and helped to facilitate the research process.

Conflict of Interests: The author declared no conflict of interests.

\section{REFERENCES}

Anderson, L. (2004). "Implications of Psychiatric Disorders during Pregnancy and the Postpartum Period - A Population-based Study.”UMEA UNIVERSITY MEDICAL DISSERTATIONS, New series, No 926, ISBN 91-7305-750-9 ISSN 0346-6612.

Čuržik, Doris \& NatašaJokićBegić (2012).“The Utility of Bdi-Ii in Assessment of Pre- and Postpartum Depression Symptoms and Their Relation to Labor Pain."Psychiatria Danubina, 24(2), 167-174.

Fisher, J., Cabral De Mello, M., Patel, V., Rahman, A., Tran, T., Holton, S. \& Holmes, W. (2012)."Prevalence and Determinants of Common Perinatal Mental Disorders in Women in Low- And Lower-Middle-Income Countries: A Systematic Review.” Bulletin of World Health Organization,90, 139-149.

Gebuza, G., Kaźmierczak, M., Mieczkowska, E., Gierszewska, M. \&Kotzbach, R.(2014).“Life Satisfaction and Social Support Received By Women in the Perinatal Period.”Advances In Clinical And Experimental Medicine,23(4), 611-9.

Gerald, L. Klerman(2006). "Dr. Klerman Replies." American Journal of Psychiatry,140(5), 650B-651.

Goyal, D., Gay, C. \& Lee, K.A.(2006).“How Much Does Low Socioeconomic Status Increase The Risk of Prenatal and Postpartum Depressive Symptoms in First-Time Mothers?” Women's Health Issues, 20 (2), 96-104.

Mina, S., Balhara, Y.P.S., Verma, R. \&Mathur, S.(2012).“Anxiety and Depression amongst the Urban Females of Delhi in Ante-Partum and Post-Partum Period.” Delhi Psychiatry Journal,15, 347-51. 


\section{Depression during Pregnancy and Postpartum Period among Women}

O'Hara, M. W.(1986).“Social Support, Life Events, and Depression during Pregnancy and the Puerperium.”Archives of General Psychiatry,43, 569-573.

Otchet, F., Carey, M.S., Adam, L.(1999).“General Health and Psychological Symptom Status in Pregnancy and the Puerperium: What Is Normal?”Obstetrics and Gynecology,94(6), 935-41.

Paykel, E. S. (1991). “Depression in Women.” The British Journal of Psychiatry, 158 (Suppl 10), 22-29.

Seligman, M.E.P. “Fall into Helplessness.”Psychology Today (1973); 7, 43-48.

Upmanyu, V.V. \& Reen, M.(1991)."A Comparison of Married Employed and Unemployed Women on Depression Measures.”Journal of Studies,4, 153- 162.

Zung, W.W.K. (1965)“A Self-Rating Depression Scale.”Archives of General Psychiatry,12, 6370.

How to cite this article: Kumari P, Raina G (2017), Depression during Pregnancy and Postpartum Period among Women, International Journal of Indian Psychology, Volume 4, Issue

2, No. 86, ISSN:2348-5396 (e), ISSN:2349-3429 (p), DIP:18.01.038/20170402, ISBN:978-1365-68609-2 